\title{
Risk Management Regime and Its Scope for Transition in Tokyo
}

\author{
Maiko Nishi*,\|, Mark Pelling ${ }^{\dagger}$, Masumi Yamamuro*, \\ William Solecki ${ }^{\S}$ and Steven Kraines \\ ${ }^{*}$ Columbia University, USA \\ †King's College London, UK \\ The University of Tokyo, Japan \\ ${ }^{\S}$ University of New York, USA \\ "Tokyo City University, Japan \\ Imn2626@columbia.edu
}

Published 2 February 2017

\begin{abstract}
Coastal megacities are highly vulnerable to climate change due to asset concentration and hazard exposure, but have potential for innovative risk management taking advantage of technological, economic and political capacities and cultural assets. Tokyo is the center of one of the world's largest urban agglomerations and one of the most hazard prone global cities. Having experienced repeated extreme events and resultant devastation, Tokyo has deployed a strategy of high technology based risk management. In the face of climate risks that amplify ongoing threats from catastrophic earthquakes, it is unclear whether the current strategy and its attendant culture and administrative structures are an enabler or a barrier for climate change adaptation. Based on 24 expert interviews, this paper examines Tokyo's readiness to transition from its current risk management orientation aimed at disaster prevention towards more resilient of transformative states. We find the current risk management regime has been moving towards resilience planning promoted by the national policy architecture and the leadership of the Tokyo Metropolitan Government by incorporating self-help and community-cooperation into a long-standing strategy of resistance. This strategy continues to be dominated by technological, rather than social policy and so misses an opportunity for a broader contribution to sustainable development. The current approach may work for the near future but is perhaps less well suited to long-term risk management which includes highly uncertain future climate risks and potential social
\end{abstract}

"Corresponding author.

This is an Open Access article published by World Scientific Publishing Company. It is distributed under the terms of the Creative Commons Attribution 4.0 (CC-BY) License. Further distribution of this work is permitted, provided the original work is properly cited. 
change. Transition is impeded by structural bottlenecks in the city authority while strategic partnerships between different stakeholders can facilitate transition of public values. Realizing this flexibility will position Tokyo's risk management regime to better play a role in longer-term sustainable development.

Keywords: Climate adaptation; Risk management; Transition; Tokyo; Sustainable development.

\section{Introduction}

Tokyo is one of the world's largest urban agglomerations (UN 2015). At its center is the jurisdiction of the Tokyo Metropolitan Government (TMG). TMG, a political center as the national capital, administers a population of 13.6 million (TMG 2016a) and a growing share of Japan's national economic activity (Sassen 2013). At the same time, since its founding Tokyo has been one of the world's most hazard exposed cities having experienced repeated devastations associated with earthquakes, fires, aerial bombings and other hazards over the past four centuries (Seidensticker 1983, 1990). Evolving from disaster management combined with infrastructure development, particularly since the Great Kanto earthquake and fire of 1923, Tokyo has developed a sophisticated risk management system equipped with "an arsenal of engineering technologies" (Mitchell 1999: 56). This was already observed by the end of the last century in the face of a series of urban challenges such as high land costs, substandard housing, traffic congestions, terror gas attacks and the potential for a major earthquake anticipated in the near future. The system featured not only the use of high-technology instruments to deal with high-impact natural hazards, but also a centralized decision-making process with "widespread public acceptance and cooperation" (ibid. 57), with earthquake risk as its primary concern.

The centralization of Tokyo's risk management regime is not unquestioned (Aldrich 2012, 2013). Longstanding struggles between citizens and the state, have placed activists including NGO leaders, scientific experts and young liberal politicians in leadership roles for civil society (Avenell 2016; Aldrich 2008; Hasegawa et al. 2007). The 1990s were a particular period of civic resurgence met by state cooption (Ogawa 2004). Since this period, bottom-up, community-based approaches for risk management and wider development agendas have become more prevalent and gained government support having proven themselves efficient in disaster reduction and recovery (Aldrich and Meyer 2015; Aldrich and Sawada 2015; Aldrich 2016; Nakagawa and Shaw 2004). The so-called '3/11' disasters following the Great East Japan earthquake, tsunami and nuclear incident in 2011, quickly led to a popular questioning of the role of central government, science, corporate private sector interests and citizens in strategic decision-making for risk management and its relationship with development. However, such questioning of 
fundamental roles and responsibilities soon gave way to a more superficial but pragmatic mobilization around disaster risk volunteering, citizen science. This has helped to build increased trust between citizens (Aldrich 2012, 2013; Veszteg et al. 2014; Hommerich 2012). Thus, despite the expectation of the post 3/11 Japan to break away from top-down, technocratic decision-making processes evidence shows limited institutional change towards a more inclusive national institutional framework (Samuels 2013; Aldrich 2011, 2016; Avenell 2016). If, as it seems, the post 3/11 era regime in Tokyo is rejecting fundamental transitions how fit is a still centralized and techno-centric risk management regime for anticipating the risks of climate change extremes?

City-level responsibility for managing the adverse effects of climate change gained momentum following Local Agenda 21 in 1992, and further the amendment of the Act on the Promotion of Global Warming Countermeasures in 2008 (Onishi 2010). Most attention to date on Tokyo's climate change governance has focused on mitigation (e.g., Aoki and Motoki 2007; Aoki 2010; Baba 2010; Oono 2010). More recently, adaptation to climate change has gained currency in the scientific and policy arenas in Japan. While the National Plan for Adaptation to the Impacts of Climate Change was released in November 2015 (Government of Japan 2015), formal policy mechanisms at the local level are still in development. A handful of studies have evaluated local adaptation strategies in Japan (Tanaka et al. 2011; Shirai and Baba 2014; Shirai and Tanaka 2015). Yet, it remains unclear how climate change adaptation is prioritized in the overall context of risk management and to what extent the city incorporates future climate risk management in pursuing its development goals.

Tokyo continues to be in peril of a possible powerful inland earthquake and other urban hazards. Whereas the risk management system has been continuously framed by these extreme events, it is unclear how far current governance culture and structures enable or constrain adaptation to climate change extremes. Both vulnerability and hazard are emergent properties and less prominent in Tokyo's historically oriented risk management vision and planning. Risk in Tokyo continues to be overwhelmingly understood through historical antecedents - less through future oriented risk modeling. This arguably distorts the policy balance between climate and other hazards and makes difficult a clear vision for adaptation policy and the role of development priorities in generating or reducing vulnerability and risk.

This paper investigates challenges and opportunities for adaptation in Tokyo as a coastal megacity. Analysis draws on 24 key stakeholders interviews and respondent views on how Tokyo might best position itself to manage future vulnerability to climate extreme events such as heat waves and flooding. The study explored the following questions: 
(1) How does the city approach urban hazard and associated risks?

(2) What is the current risk management regime particularly applied to heat wave and flooding?

(3) What scope is there for transitioning the regime to be better positioned to manage future development and risk contexts?

The next section introduces the methodological framework. The subsequent section presents results and discussion which are summarized in conclusion to draw out primary elements of Tokyo's contemporary risk management regime status, potential for and any observed direction in transition.

\section{Theoretical Framework and Methods}

A risk management regime is an established set of institutions, norms and behaviors that dominate a pattern of risk management, wherein a set of assumptions and assertions concerning the conceptualization of risk and the roles and responsibilities of various governance structures are embedded (Solecki et al. in press). To describe the process of regime change, we use the transition framework (Solecki et al. in press). The transition framework outlines four states of risk management regime: collapse (no strategic planning), resistance (planning for stability), resilience (flexible planning) and transformation (planning for fundamental change). In particular, our study distinguishes the four regime states resting on different aims: (1) to provide stability for planning and business so that development is not interrupted by risks or risk management (resistance); (2) to help planners and business learn how to accept some impacts of hazard risk in order to avoid major losses (resilience); (3) to change current planning and business practices that are seen as being unfitted and as causes of risks (transformation); and (4) to have no strategic policy, where any activity is highly fragmented and uncoordinated (collapse). With this framework, the study seeks to identify the current risk management regime for Tokyo and its limitations and potentials for transitioning towards alternative states for urban coastal adaptation.

Data were collected in August 2015. Interview schedules followed a design common to aligned studies being undertaken in Kolkata, Lagos, London and New York (see bel-truc.org). Semi-structured interviews were conducted with 24 urban risk management experts identified through snowball sampling that began with academic contacts and a multi-stakeholder network of the R!SE initiative engaged in disaster risk-sensitive investment. The sample consisted of: 14 academics; 2 TMG officials, 2 national government officials, and 6 private-sector experts. The study was constrained by respondent access which was skewed towards academics involved as advisors or consultants in city risk management 
planning and practice. This has influenced the findings which emphasize sciencepolicy relations in processes that drive or constrain innovation in planning for risk management.

\section{Results and Discussion}

\subsection{Political priorities across urban hazards}

How does Tokyo approach climate risk management in the face of various urban hazards, interests and needs? The overarching policy goal of TMG under the Masuzoe administration (2014-2016) was officially expressed as Tokyo, the world's best city with emphasis on three themes: welfare, economy, and the 2020 Summer Olympics (TMG 2014a). As one of its eight Strategies, the 'Safe and Secure' Strategy uses the terminology of resilience (e.g., to ensure a high level of disaster resilience) (TMG 2014a). Yet, operationally, the Strategy largely involves resistance-type measures characterized by fireproofing, and the seismic and water resistance of infrastructure to protect existing assets, to which resilience mechanisms of self-help and mutual assistance are attached as secondary mechanisms. Provided with the largest budgetary share among all eight strategies (more than one third of the total budget for the first 3 years), considerable financial resource has been funneled into risk management. This has been oriented to a range of hazards including earthquake, torrential rainfalls, landslides, street crime, cyber-attack and organized crime (TMG 2014a).

Budget share gives some insight into the prioritization of each hazard type though risk management costs depend on the type of hazard and its countermeasures (e.g., often a larger cost for infrastructure development than public relations), while the effects of a policy may overlap between different hazards (e.g., urban renewal projects addressing both fire and earthquake). Nevertheless, the budget breakdown for the fiscal year of 2016 shows higher investment in fire and earthquake, followed by coastal and rainfall flooding and then heatwave, with much less investment into street crime and terrorism (TMG 2016b). ${ }^{1}$ This is

\footnotetext{
${ }^{1}$ Accounting for about one-fifth of the total budget for the major policies, the budget for safety and security (JPY 346.2 billion, approx. US\$ 3.3 billion as 100 percent) is broken down into measures addressing: (1) fireproof and quake-proof of crowded blocks of wooden dwellings (27 percent), (2) heavy rainfall (19 percent), (3) tsunami and storm surge (15 percent), (4) empowerment of communities (14 percent), (5) quake-proof of public buildings (11 percent), (6) roads for emergency transportation (10 percent), (7) street crime (1.6 percent), (8) terrorism and cyber-attack (0.8 percent), and (9) disaster medical care ( 0.3 percent). In addition, as part of the budget for long-term city development beyond 2020, a budget of JPY 17.7 billion (i.e., equivalent to about a half of the above budget for roads for emergency transportation) is allocated for measures against global warming and the heat island effect.
} 


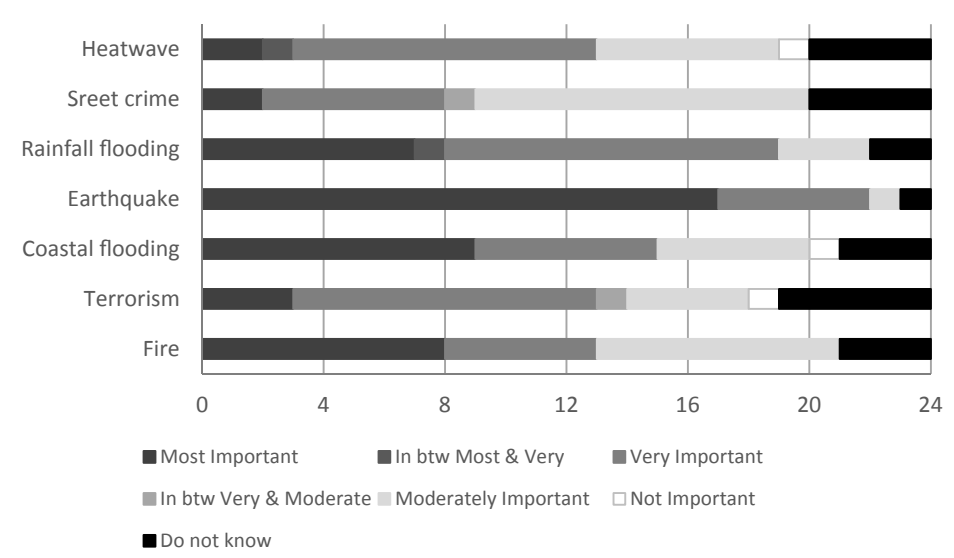

Figure 1. Importance of Hazards (Policy Priorities)

consistent with respondents' views of city priorities (Figure 1) and largely with their own professional judgments (Figure 2), indicating consistency in public and technical discourse. Exceptions are heatwave, and coastal and rainfall flooding. Some 25 percent of respondents $(n=6)$ perceived less policy priority given to heatwave than their own judgment, and one forth $(n=6)$ and one fifth $(n=5)$ of respondents saw less policy priority given respectively to rainfall and coastal floods, leading respondents to express concerns for the regime's strategy for climate risk. In particular, many emphasized the exposure of areas below sea level to coastal flooding. One challenge for Tokyo is to shift from an earthquake centric to a fully multi-hazard approach to risk management.

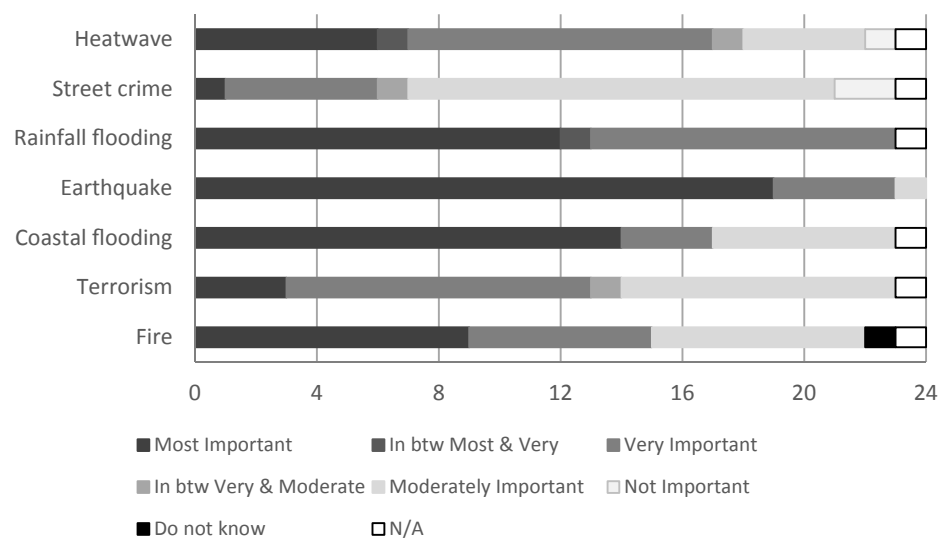

Figure 2. Importance of Hazards (Professional Judgment) 
TMG has no official climate change adaptation policy. Although Hijioka et al. (2016) argue that 44 percent of standing TMG policies can be described in terms of climate change adaptation, these have not yet been brought together under a coherent policy regime. Our interviews revealed that policy considerations concerning climate change adaptation have been underway at TMG since their commitment to the C40 Tokyo Conference held in October 2008. The slow speed of policy development was explained by the long time taken for an initial study of climate change impact, the need to adjust the administrative structures at TMG following a change in leadership, and increased public concern on earthquake risk following the Great East Japan earthquake of 2011. Perhaps part of a reluctance to plan for future risk is based in current framings of risk which are grounded in historic - not projected - extremes. TMG continues to review and upgrade measures against flooding and heatwave based on recorded extremes in river flow and urban temperatures. In this connection, a senior manager at an environmental division of TMG stated:

Having observed the increased frequency of intense rains exceeding $100 \mathrm{~mm} / \mathrm{h}$ falls over the preceding 30 years, we developed the "Small and Medium River Maintenance Policy" in 2012 where the target rainfall was raised from $50 \mathrm{~mm} / \mathrm{h}$ to either $65 \mathrm{~mm} / \mathrm{h}$ or $75 \mathrm{~mm} / \mathrm{h}$ depending on the areas. The previous target was determined based on the estimate that the Isewan Typhoon in 1959 was likely to be the largest typhoon into the future. But the trends have changed, and accordingly the target was raised. The frequency and intensity of rainfalls may change in the future, but we have not yet taken into account such future risk change in developing plans and policies. ...It is what we do as usual that we develop and implement our interventions based on the records with the assumption that climate trend is more or less stable.

\subsection{Current risk management regime}

Respondents characterized the contemporary climate risk management regime for Tokyo as approaching an inflection point, moving from resistance to resilience (Figure 3). Resistance was illustrated by Tokyo's pursuing risk management largely through the accumulation of infrastructure. Some respondents saw this as a sound policy for a highly engineered megacity with expectations that public spending would continue. The emerging resilience approach in Tokyo was framed not as an alternative to the stability seeking of resistance, but as an extension 
10

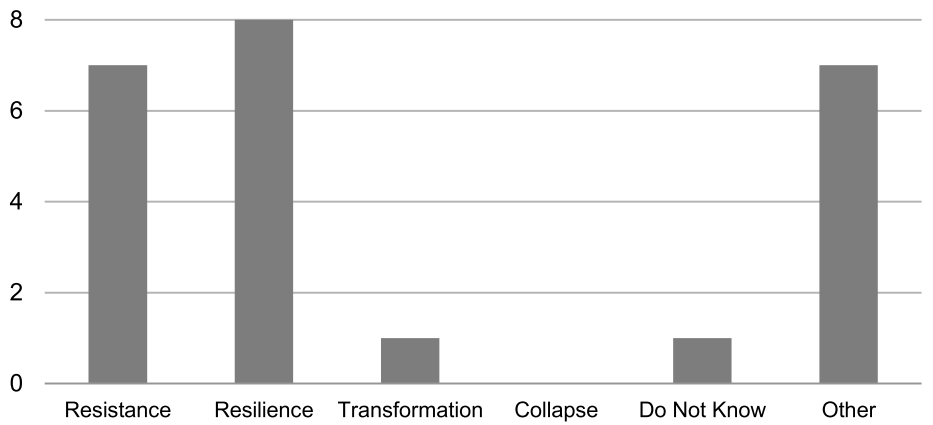

Figure 3. Dominant Approach

to resistance. Resilience was alternatively referred to as self-help (jijo in Japanese) or community cooperation (kyojo), to be prepared for risks associated with unforeseeable extreme events or future climate change. As exemplified in policy documents, TMG has increasingly promoted empowerment of individuals for selfhelp and communities for mutual assistance through disaster drills, information provision and educational campaigns (TMG 2014a). Thus, there is movement from resistance to resilience. Yet, resistance approaches remain at the base of Tokyo's risk management orientation, and are positioned to provide stability and support development, with flexibility at the level of citizens and communities rather than in business models or infrastructure planning.

This dynamism in the relationship between resistance and resilience generated some ambiguity. A respondent from the TMG agency described their current position as being one where planners were actively incorporating resilience into resistance. More emphatically, a respondent from academia with expertise in hydrology highlighted the absence of a boundary between resilience and resistance in Japanese thinking, stating:

The concept that "development not to be interrupted by risks or risk management" does not apply to Japanese words. As life [security] and economy always go along together, we don't have a sense of either life or economy. Tokyo certainly has pursued economic development as seen in reclamation land there. But development has promoted employment, and even with job boost there is no case of more than threefold income disparity between employers and employees. We have historically shared efforts and pains, and worked in collaboration. Though a lofty policy maker 
may develop a plan with clear-cut aims, people at work including government officials make efforts somberly to turn out to be both stable and flexible in balance to the fullest.

The depth of coupling between economic development and social wellbeing in Japanese consciousness and language has important implications for understanding Tokyo's adaptation-development nexus. Trading economic flexibility for social gain through risk management is less important than economic security in a context where levels of inequality and poverty are popularly understood to be low. In fact, the relative poverty rate of Japan is on the rise and has been above the OECD average since the mid-2000s, with Japan's Gini coefficient today higher than the OECD average (OECD 2014). Further, the proportion of the population living in absolute poverty increased by 5 percent between the mid-1980s and 2000 (Jones 2007). Despite this a lower proportion of the population is concerned about income inequality in Japan than in other countries with similar levels of Gini coefficient (Ministry of Health Labour and Welfare 2011). High levels of employment (OECD 2014) and equitable income distribution continuing since the post-war period have led to a long-held self-perception of Japan as an egalitarian and classless nation (Abe 2011). This highlights the stability of cultural values, but also raises the potential of a break in these values should increasing poverty and inequality continue to rise, and the implications of this process as a determinant of politically acceptable risk management strategy. Though Japan's 'rediscovery' of poverty is lagging behind its experience (Pantazis 2014: 63), it may affect popular and political thinking on the preferred balance of economic and social interests in development and their expression in risk management. As discussed later for future trajectories, it might not be beyond imagination that emphasizing resilience making to be the responsibility of vulnerable populations - who may also be increasingly seen as unequally poor - is less tenable in the future, or will require stronger state support as a mechanism for wealth redistribution, for example through a review of social or property insurance policy. Social transformation outcomes of risk management that act to correct development injustices as a means for and outcome of reducing vulnerability may become more politically attractive in this future.

\subsubsection{Drivers leading to the contemporary regime}

Respondents reported that the most important elements contributing to the shaping of the contemporary regime included science and technology, and national policy steering through legal jurisdictions and budget allocation. As shown in Figure 4, respondents pointed to hazard information as an essential element for policymaking. At the same time, as seen in Figure 5, the national government has had a 


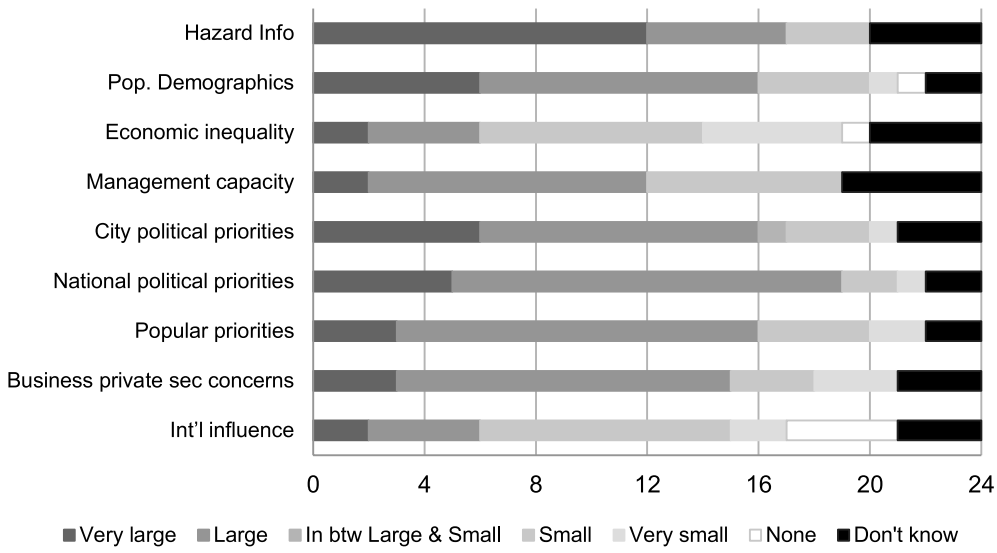

Figure 4. Factors Leading to City Policy Priorities

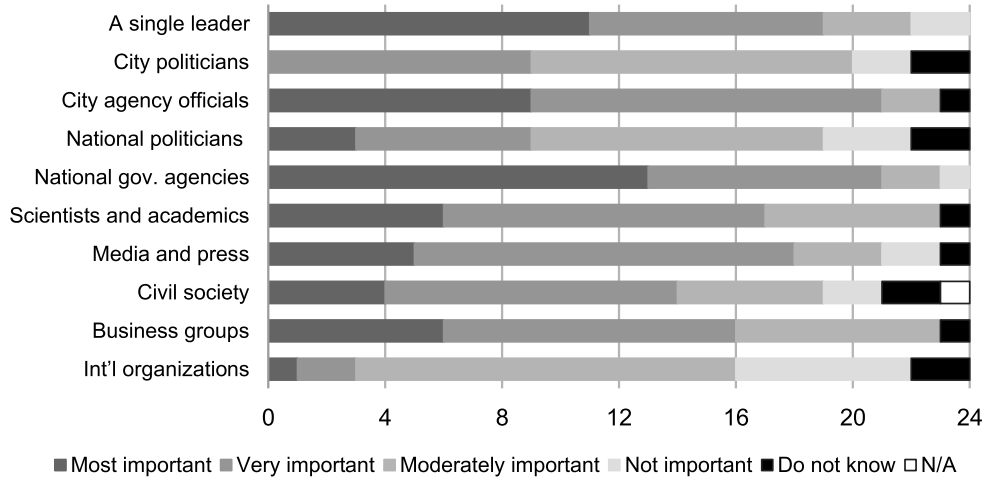

Figure 5. Actors Establishing the Dominant Approach

considerable top-down influence on city policy through national policies, legal systems and budgetary procedures, wherein knowledge held by academia, as well as public opinion influenced by business concerns and popular priorities, were drawn on for policy-making. A national official in the water sector highlighted TMG's role as a city manager, through which the national government has mainstreamed the resilience approach using legal reform:

The mainstreaming of resilience is underway at the national level, as seen in the recent legislative changes. It is the massive tsunami following the Great East Japan earthquake that served as the impetus for current movement. We recognized that such a giant tsunami can actually occur even though neither anyone had experienced nor any historical record had mentioned it. In response, 
the national agencies together with academia have been discussing and creating a movement for a resilience society. For legislative change, national politicians have also worked with us. In addition, other stakeholders are all important, allowing us to introduce new policies to society.

The high priority given to (earthquake) risk management in popular and political discourses meant there was no discernable, individual political party leadership on this issue - all agreed it was important and none had yet concretely extended from earthquake to climate change hazard agendas. Individual leadership, however, did make a difference in TMG: the Governor of Tokyo leads TMG and influences its strategy, and to some extent Metropolitan Assembly members who influence specific measures, but not overall TMG strategy. A senior manager at TMG pointed out that the Governor served as the ultimate decision maker in policy making, with city officials playing a role as technicians and administrators in policy development and implementation:

As the governor does not always have knowledge and information across all the different sectors and fields to make a final decision, it is the officials who offer well synthesized and timely information, including suggestions of when and what issues should be prioritized and undertaken. For example, press launches for new strategies are presented in the presence of the governor. It is the governor who has the last word, and the press launch is influential only because the governor is present. There are also, but not so often, very informal cases that officials draw on support from city politicians to pursue some measures for goodwill depending on the circumstances.

Mirroring national political culture, TMG is a centralized authority but one that draws in selected external contributions. Reflecting on the evolution of the risk management regime a national official of the environment sector described the process as experience-based and bottom-up referring to environmental policies in Japan as continuously responding to emerging environmental and social problems. Responsiveness led an academic architect to describe policy formation as muddling through, in which policy was decided in response to the emergence of problems reflecting public opinion. These views confirm studies highlighting the limited opening of spaces for external influence and the contribution of civil society actors in shaping public discourse on specific risk issues such as nuclear power development and environmental pollution (Hasegawa et al. 2007; Avenell 2015, 2016). 
Despite legal support for decentralization, including change to electoral institutions in 1994, the passage of the Non-Profit Organization Law (NPO Law), 1998 and the Decentralization Law, 2000, the risk management regime remains largely technocratic and top-down rather than innovative and proactive (Aldrich 2016). A senior manager at TMG summarized the common practices of policy making at TMG:

Objective information such as hazard information, demographics and economic status is always analyzed and incorporated into policy making. Given analyzable objective information, political will essentially drive policy making. In addition, the city agency's capacity determines whether policies can be implemented and enforced, while the governor makes a decision also referring to the citizens and the metropolitan assembly. National political priorities do not directly drive local policy making, but control it through budget allocation. Business and private sector concerns either directly affect the governor's will or indirectly influence it through national political priorities. There are few cases of external pressure from the international community influencing disaster management in Tokyo.

The centralized national and TMG regime was critiqued by respondents from academia who raised concerns over the transparency and credibility of policymaking this brought. In particular, although scientists' direct participation in discussion sessions were sometimes drawn on for policy deliberation, a few respondents questioned the degree of influence and legitimacy held by scientists, suggesting that their participation may only give cosmetic legitimacy to ongoing policy processes. A respondent from academia commented:

It is not easily visible from outside the agency how local policy priorities are actually decided. I guess TMG may have a strong will to pursue policies not necessarily following the national government. However, the contents of the policies seem to be determined according to the opinions of the people with the loudest voices at the time such as prominent scholars in a way that maintains the TMG's overall direction or independence. For example, if social awareness of heat grows stronger, related studies will increase and then TMG will take an initiative to develop countermeasures against heat island effects. When it comes to what measures should be taken, they will develop the 
measures ... exactly following what a prominent scholar suggests. But there are often cases that actual validity and justification of such measures are unclear or questionable.

The response from TMG explained the rationale behind this opaque process, describing that the city government often internalized or informalized policy-making processes for efficiency and effectiveness in the face of budgetary constraints and the traditional hierarchical structures of academia. On one hand, 'informal' study meetings with experts from government, private sector or academia allowed for exchange and supported innovation. On the other, there was a strong cultural preference for respecting existing academic hierarchies when engaging with science, limiting the diversity of science voices. The TMG also commissioned private consultants or research institutes to draw on external intellectual resources where TMG saw a specific need. But the choice of consultants was seen to be based overly on price rather than quality, which may be hard to judge in areas of emergent policy. Together these administrative and cultural factors produced a policy environment that while based on trusted expertise was less adept at surfacing emerging ideas to inform policy deliberation, this may serve contemporary risk management well (based on historically grounded earthquake risk) but is of more questionable utility when planning might also require a future lens to capture Tokyo's emergent multiple hazards and social change.

\subsection{Future trajectories in risk management}

Finally we ask, what management approach could the city deploy to address future climate risks? First, to explore future risks, respondents were presented with four scenarios that might emerge over the next 20 years (towards 2035). These were: (A) a doubling of the current frequency of climate hazard events (i.e., flooding and heatwave) and their impacts; (B) a doubling of the population over the age of 65 in addition to doubling climate hazards; (C) a doubling of the level of income inequality in addition to a doubling of the aged population and of climate hazards; and (D) halved resources expended per capita on disaster risk reduction in addition to a doubling of inequality, the aged population and climate hazards.

Scenarios were first discussed for relevance (Figure 6). Population aging was perceived as most likely to inform policy. Given the positioning of inequality (and poverty) as triggers for regime change in risk management, the belief that inequality was unlikely to increase in the next 20 years inferred a belief also in stability in the policy regime. This said, decline in government capacity and somewhat the progress in population aging were signaled as most likely to lead transition towards transformative regimes where development policy would itself 


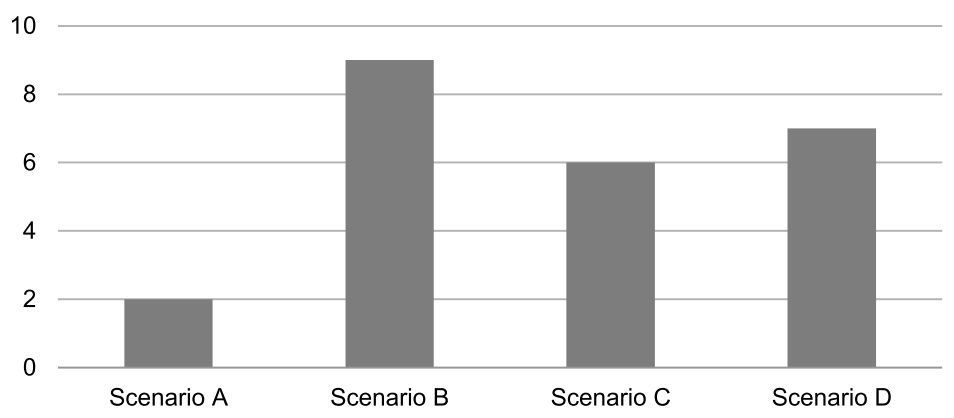

Figure 6. Most Plausible Scenario

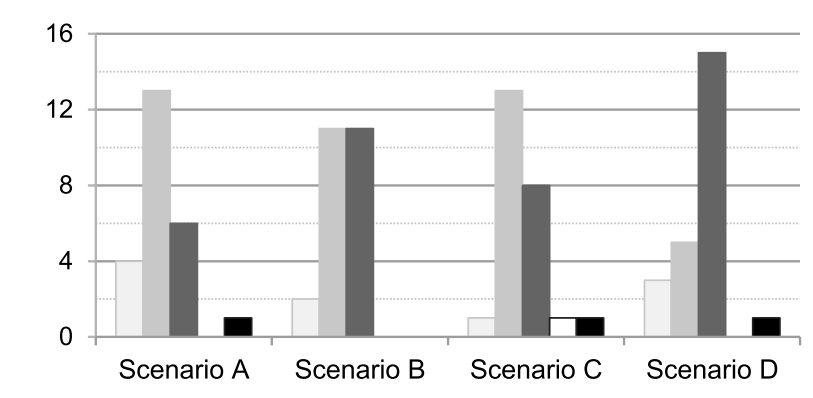

$\square$ No change $\square$ Minor change $\square$ Major change $\square$ Other $\square$ Do not know

Figure 7. Degree of Change to Be Made

be a target for risk management. Figure 7 shows the importance of social dynamics in forcing a transition. Respondents felt a doubling of hazard could be dealt with through minor adjustments to the existing regime, but any large increase in vulnerable populations would require a transition in risk management.

Tokyo does face an aging population, it is estimated that one in three citizens in Tokyo will be over the age of 65 by 2035 (with an increase from 24.2 percent in 2015 to 30.7 percent in 2035) (TMG 2015). TMG has taken aging into account in its existing policies, encouraging some respondents to suggest little change would be required in the future. For around half the respondents, however, more radical changes were suggested including the need to develop compact city or national land plans to optimally locate the aging population to secure public services provision. Others proposed a political decision to determine an acceptable level of risk associated with aging indicating the ethical and value-judgments needed. In particular, an expert in medicine commented:

We very often accept elderly critical-care patients suffering from heat disorder and any other diseases. ... What would be the best 
solution to the elderly and who should decide it? Their family usually want the elderly to be saved, but it may not be possible for them to recover 100 percent. The urban environment does not allow the elderly to be appropriately cared for at home, as housing is small and family members are busy with their own jobs.

While outside the scope of the presented scenarios, a number of respondents volunteered concerns about the opportunities and threats of migration as a response to aging (the potential of securing a workforce set against possible cultural tensions), and that this may be more important than aging as a driver for demographic change. For some respondents both population aging and economic stagnation were plausible in the coming decades, potentially threatening stability in government. Under this interpretation, many respondents called for transition. Suggested changes included innovations in the means for self-help and community cooperation such as non-residential zoning and relocation from hazardous areas, private insurance, new living arrangements including shared accommodation. Some even considered large-scale relocation of core Tokyo functions. Several respondents raised arguments for deeper transition in policy making processes and political structures: a respondent from academia argued that such a scenario would cause the opening of a public debate on minimum standards of social wellbeing with potentially transformative consequences for risk management and development in the city, and beyond:

If economic inequality significantly grows, there may be some people who cannot afford an air conditioner even when the AC installation is promoted. If so, we will need a societal consensus about what society Japan seeks to attain. As for the right to life guaranteed in Article 25 of the Constitution of Japan, we will need to specify what kind of living is the minimum. Our awareness of a minimum standard was not high when Japan was an economic power with a fat tax revenue, but I don't think the society will tolerate any further the cases that people receiving welfare have more income than workers earning minimum wage. ...Neither minor nor major change may be meaningfully made unless the contents of the right to life are well discussed. ...If the government capacity largely diminishes additionally, the soft approach involving the change in public values will be further more critical. As examples of self-help, people are only encouraged not to live in a dangerous location or to purchase insurance for their own risk management. 


\subsubsection{The emerging direction of change}

With the above future risks in mind, the majority of respondents perceived the current direction of transition in the risk management as moving further towards resilience (Figure 8). Some foresaw continuation of efforts to mainstream resilience into disaster management, under the assumption that political structures and financial constraints would make difficult any deviation from support for resistance. Others pointed to the increasing emphasis on flexibility at the individual and community levels to prepare for unprecedented disaster risk as an indicator that resilience was gaining momentum. In either case, transition was not seen as breaking away from economic stability as the primary goal for risk management, with the recognition that existing infrastructure should be at least maintained in the face of Tokyo's dynamic hazard risks. The primary factors shaping this transition towards resilience included extreme events and economic change enabled by administrative structures, with more than two thirds of respondents describing these three pressures as the most important (Figure 9). Speed of transition was described as slow, but consistent with transition being detected over the last 20 years.

While Tokyo's risk management has long been oriented towards resistance, its expression has changed over time and the emerging incorporation of elements of resilience in the regime can be traced over several periods. Importantly though, past reforms have tended to follow catastrophic events and not been built on future risk or sustainable development prospects reinforcing the challenge for Tokyo as it enters an era of climate risk combined with present earthquake risk. Historical precedents include response to the Great Hanshin earthquake (1995). The impacts of this earthquake influenced both the concept of administrative planning of Tokyo and the role of civic actors in risk management nationally. As part of this reform, TMG initiated 'pre-disaster' planning for the first time, putting in place planning

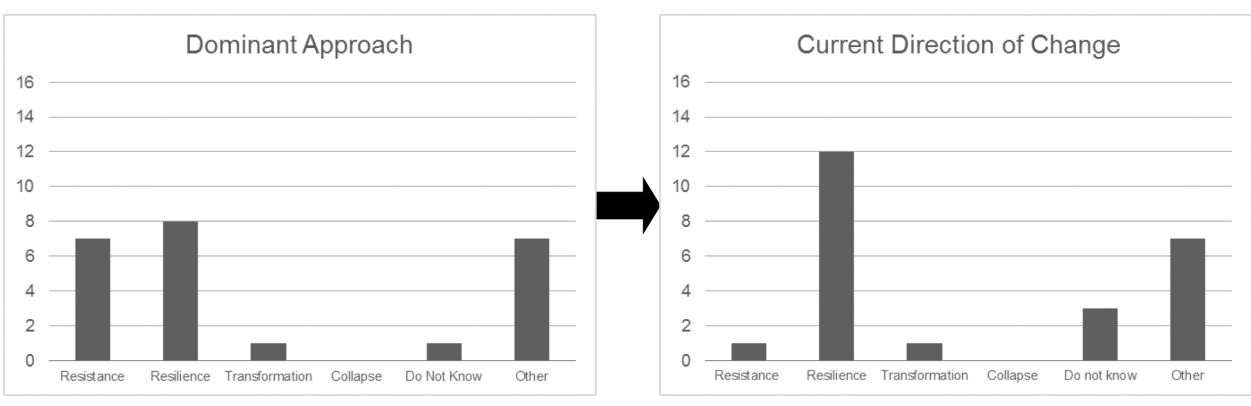

Figure 8. Current Direction of Change 


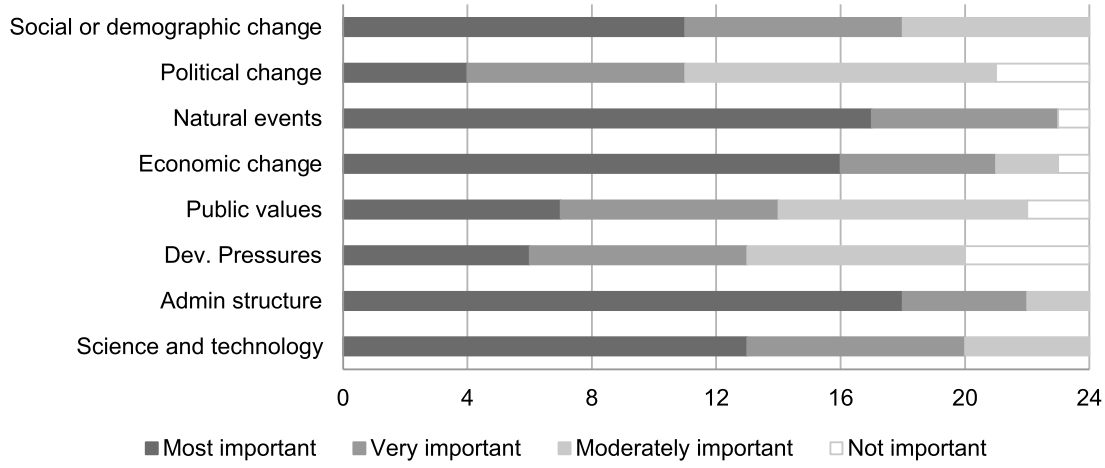

Figure 9. Factors Shaping the Current Direction of Change

and administrative manuals for post-disaster urban restoration (Aiba et al. 2007) along with plans for urban improvement focused on high vulnerability crowded wooden dwellings under the Ordinance of 2000 (Nakabayashi 2008). Also, with the lessons that about 98 percent survivors were saved on their own or by neighbors (Fire and Disaster Management Agency 2016: 262), the preamble of this Ordinance introduced to official policy documents for the first time in Japan the three-fold concept of self-help (jijo), community cooperation (kyojo) and government assistance (kojo), which has gained currency in disaster management nationwide (Iwasa 2008). These reforms show residents, residential land-use and community groups as the target for reform and arguably for steps towards a resilience transition with commercial and industrial behavior not addressed directly.

Business life, and especially supply chains were the target of reform following the Great East Japan earthquake and tsunami (2011). Besides the widened trust deficit between citizens and the state (Aldrich 2012), the unparalleled, extensive disasters stemming from this earthquake exposed the vulnerability of Tokyo's reliance on electric power and materials from other regions (Numata et al. 2011; TMG 2011). In particular, the disaster signaled for Tokyo the criticality of supply chains as well as severity of hazards that exceed infrastructure based on the principle of resistance (disaster avoided). In response, TMG revised the Local Plan for Disaster Prevention (2012) to include preparedness for an unprecedented large-scale disaster with emphasis on partnerships among actors (TMG 2014b). Measures for self-help and community cooperation were further strengthened for risk reduction, and also continuity planning in government and business sectors. 
Historic climate extremes have also begun to act as drivers of change in risk management and its relationship to development. Several large-scale floods caused by unusually intense rainfall have led to increased standards for flood control, monitoring, information provision and evacuation processes. The torrential rainfalls in summer 2008 served to coin the term "guerrilla storms" (gerira gou), and led to improved precipitation monitoring for faster and more accurate information provision for individuals. Flash flooding in Tokyo in 2008 gave rise to earlywarning operational process at sewerage construction sites at TMG. International processes also influenced TMG's risk management as TMG embarked on policymaking for urban heat island effects and global warming as a 'double warming,' following Japan's agreement to the Kyoto Protocol (1997). Yet, more substantively, recent hot summers, particularly the high death toll nationwide (1,745 fatalities) associated with the extreme heat in 2010 (Ministry of the Environment 2014), were perceived to have led to a departure from earlier government-led measures such as roof greening incentives, towards a preference for measures aimed at directly managing heatstroke. In this regard, a respondent from medicine described:

Although the death toll from heat disorder had never exceed 1,000 fatalities before 2010 even during the extremely hot summer in 2007, it reached 1,700 fatalities in 2010. This was a shocking event. In the following summer after the nuclear explosion in Fukushima in 2011, we were worried about numerous fatalities under the planned saving electricity, but the death toll turned out to be below 1,000 because of the cooler summer. Yet, since this time around, we have become busy and the initiatives to tackle heatwave have been actively promoted. I guess we have come to notice symptoms, as we recognize much hotter in summer today than 10 years ago, obviously feeling stronger sunlight. ...Rather than the public authority, grassroots actors such as private doctors, care staff, civil society, ... and of course including public healthcare facilities and public doctors, have been increasingly acting on this with better capacities. We see better diagnostic and therapeutic capabilities of these actors, and increased citizens' knowledge of expedient treatments in the past couple of years.

This statement underlines the capacity of local actors that have been given increased responsibility to manage risks, but limited scope to inform policy. Administrative structures have been formed in response to, not in anticipation of 
such extreme events. An academic emphasized the historical context that gave rise to contemporary policy-making culture:

In Japan, things tend to be decided by government leadership, while citizens tend to place their expectation with the government. Compared to Western cities, citizens' sense of participation in politics may be lower. This may be in part due to the historical context that Japanese cities were not created by citizens, but rather they gathered around people with authority. Even today local residents protest against the adverse effect of a high-rise building on scenery only when such a building is about to be constructed nearby, but normally they tend to see the government as being supposed to manage the creation of scenery and it is not the place of the citizens to raise their voices. The government has the awareness needed by this role to handle a matter before problems emerge, and generally citizens have no question of there being any problem in policy making under government leadership.

Within this context, administrative agencies were largely seen as responsible, interacting with economic and business activities (that were coupled with social wellbeing) and with support from science and technology to progress disaster management. Despite a lack of transparency in mechanisms for integrating scientific knowledge into policy-making, the response of TMG officials suggested that scientific information was a major input in policy development. The use of science has been strengthened following the call from the Sendai Framework for Disaster Risk Reduction 2015-2030 for an 'evidence-base' and 'improved understanding' of disaster risk in national policy. Albeit still largely a government-led regime, a more inclusive approach involving the general public has been promoted following the heightened public attention to self-help and community cooperation in the aftermath of the 3/11 disasters.

The engineering solutions of disaster management encouraged economic development in the post-war years (e.g., dams and levees) but these physical structures have now begun to decay and come themselves to pose threats to the city. For example, a roadway accident in the Sasago Tunnel, 2012, resulted in nine fatalities. This event increased attention to dilapidated infrastructure nationwide, and led to disaster management being better integrated with the maintenance or renewal of decaying infrastructure. More general physical infrastructure of the post-war years (e.g., mass housing, and water supply and sewerage systems) also limits risk management options, for example by making difficult the relocation of vulnerable residential districts or the creation of an evacuation space in Tokyo. 
Contemporary economic stagnation was also seen as a potential driver that could contribute to a shift in risk management from resistance towards resilience. This may happen if public spending for disaster management was reduced with policy shifting responsibility for adaptation further onto individuals and communities. The threat is real enough for Olympics risk management planning to have an enhanced element of self-help and community cooperation. Specifically with the prospect of the 2020 Olympics, the Tokyo Disaster Prevention Plan was published in 2014 to speed up pre-disaster planning including a potential Tokyo inland earthquake and wind and flood damage (TMG 2014c). This plan also placed an emphasis on individual behavior and community resilience building on direction of change initiated in the post 3/11 decade.

\subsubsection{Challenges and opportunities for transition}

Tokyo's risk management regime deploys social resilience to enhance the resistance to risk of economic activity. What challenges might Tokyo face if transition towards a more resilient or transformative regime were preferred? A major challenge highlighted by interviewees was the incorporation of future reforms into planning and policy-making at TMG. Elaborating on uncertainty associated with climate risk a respondent from academia noted:

One of the major challenges in risk management is the fact that there is no way to validate whether a certain plan actually gives an effect. We can know the effect only after risk becomes turned into reality. We can demonstrate possible effects only by a drill at best... But such drills are rarely conducted appropriately. In conventional environmental management, the effect can be confirmed through the Plan-Do-Check-Act cycle and thus a plan can be improved. However, for risk, the PDCA cycle does not apply. Some types of risk that more frequently become reality are more easily recognized, while others rarely actualized or not easily measurable in potential damage are less recognized. It is very difficult to deal with the latter types.

Challenges to transition were observed in standing administrative structures. A respondent with expertise in economics highlighted the desirability of a more joined-up and prevention oriented mission for risk managers:

To govern risk resulting from interactions between hazard, exposure and vulnerability, there should be multiple layers of players even in one agency. Some experts scientifically assess 
hazards, some evaluate risks based on data of hazards together with others related to exposure and vulnerability, and some allocate budget or issue evacuation orders taking into account future or immediate risks. These different players are expected to systematically engage in different functions, while there could be a position that considers all the functions and strategize comprehensively. Besides, a channel to reflect on citizens' needs and expectations is to be secured. Some tasks of hazard assessment may be outsourced to a research institute, but the government should be clear about roles and responsibilities... .There is a position of crisis manager at most prefectural governments with a focus on initial reaction to emergency situations, but no ex-ante vision overall to reasonably allocate budget and prioritize different risks.

For transition, integrated policy-making structures were seen as a key to cope with emerging uncertainty presented by climate change. A respondent from TMG indicated scope to learn from the experience of climate change mitigation planning under a former Environmental Executive that instituted agency-wide, cross-sector meetings to discuss emerging environmental problems such as heat island effects. This practice was unusual across local governments in Japan, but has been conducted somewhat as a norm at TMG. Beyond this innovation, day-to-day operations were considered to be constrained by a lack of integration reinforced through relatively rigid budget allocation. As another respondent from TMG stated, this routine practice is unlikely to lead to comprehensive long-term policy development to prepare for future risks unless a Governor's command is delivered, thus lockingin TMG to top-down cycles of innovation that may fail to move operational levels:

When the governor does not focus on climate change, the executive officials are unwilling to give themselves up to the issues that seem not to be well received. Risk of failure is larger and the response is not an easy one. So, a bar is set high for all the bureaus to take an initiative for adaptation. It is easier when there is a command from above, but we need to take our own career risk if we take own initiative to appeal to the governor. If it fails, we need to take all the responsibilities, so no one is willing to do so. No one thinks there is no need for adaptation, but as uncertainty associated with climate risk is very high, we cannot help but be wary of developing a plan particularly for infrastructure that 
requires large budget allocation, without knowing when and how much impact emerges.

Structural difficulties for innovation emerging from within TMG were further challenged by regular personnel rotation. Respondents described rotation as a practice discouraging officials from changing administrative practices as well as impeding institutional memory. Rotation creates a cadre of competence rather than excellence, and it provides an administration able to deliver but not to innovate policy. Highlighting this, a respondent from academia with expertise in civil engineering stated:

I hear often from government officials of the difficulties with lean resource allocation for infrastructure improvement for adaptation. ...Effective and efficient policy making also requires a tool to understand needs of citizens and stakeholders and continuously monitor them, but I guess the officials are not able to easily grasp such needs under the two-to-three year personnel rotation. One scholar was saying that the public administration does not create a "Superman." The regular rotation allows any official to act as a substitute, ...instead of relying on a certain Superman, so as not to centralize authority. On the other hand, such a system impedes the deepening of individual official's knowledge and the accumulation of institutional memory. There are few officials who deeply understand each policy together with the contexts and background and monitor the trends for a long term. It is often scholars, instead, who relay the knowledge continuously to new officials. As some scholars study a certain river and follow the related policies, and continuously monitor the trends, government officials interview such experts when needed for policy processes.

The responses from TMG somewhat confirmed this statement. As a norm for many local governments, three-year personnel rotation is repeated within a certain $\mathrm{Bu}-$ reau besides a temporal assignment outside the agency that is generally for five years in the case of TMG. Thus, despite a lack of continuous engagement in a specific section, officials become professionals to the extent that competence grows in a certain expert field. Nevertheless, systematic challenges in bureaucracy impede effective knowledge transfer and creation, as a respondent from TMG indicated:

To study new issues, I prefer organizing informal meetings rather than commissioning studies to external institutes, as we keep 
having a savage system of price competition for brainwork. In order to organize such informal meetings, a network of personal contacts is important, but there is no system to secure such a network. As corruption or graft is the biggest problem in government, officials are systematically prevented from being at the same position for long. Even though it is the logic for the rotation, a few key persons turn out to be more or less in similar areas continuously. Such key persons are actually critical to effectively draw on limited resources, but this is not a norm.

Mainstreaming of resilience in risk management has been promoted by national government leadership. At the local level, the resource management system (e.g., personnel rotation and budget allocation) designed to decentralize the authority appeared to impede local innovation to address emerging needs. Some respondents called for a new role of TMG in risk management. A respondent from a foundation with expertise in meteorology expressed the expectation for goal change within TMG:

We assume that the government provides administrative services for us. Such a role will remain, but their role will need to be more focused on increasing the capacity of individuals by providing scientific information comprehensively for the stakeholders including citizens. I think the government won't be able to address future challenges merely on their own. It's about time to stop saying they can provide all the services, and instead, disclose the limit of the authority and then urge people to make a decision and act on it. To do so, the government is expected to clearly and publicly explain the problems, potential impacts, response options, and scenarios if no response is taken, with concrete examples and without delay. When bottom-up capacity development doesn't work, a political decision should be required.

An official from TMG provided the view that the current government-led risk management would work over the next 20 years, given investment in highstandard engineered infrastructure. In the more distant future, however, the respondent emphasized a need for change in TMG mission making and its planning approach to address risk that may be largely different from those planned for today:

As lots of efforts and investment have been made, the current approach may work at the utmost with minor change in 20 years unless a huge, dreadful event occurs. Paradoxically it is the 
problem of Tokyo. For further future, say by 2050, ...we should be clear about how to communicate the message such that the government cannot address the problem on its own, how to make a decision on governmental roles and responsibilities, and what to do with evacuation when disaster cannot be prevented. We should start changing the approach to planning or mission making even from today, but our engineers still ought to do everything. As the traditional approach won't work further with the already negative legacy of the economy, we would need at least leadership to drastically change. The initiative should be taken by politicians, media or academia. We also talk about such a change internally, but I don't think the city agency will change from inside. ...It is actually good that the engineers have lost confidence. Previously they had been overconfident about the high-standard infrastructure, but we had the Great Hanshin earthquake and the Fukushima nuclear accident. ...It is very good we all have become unsure about ourselves and the officials have become humbled. In the field of civil engineering, academia is also hierarchical and engineers and scholars don't make an argument ... But if all feels deflated, it may change the status quo.

Respondents suggested reform could not easily be led from inside the structures of government given its jurisdictions and embedded culture. Instead, they were expectant of leaders such as the Governor or of external experts like scholars who may advise and facilitate transition to reflect external needs. Further, fundamental change in public values across stakeholders was desired. Many respondents noted the root barriers to transition in contemporary popular and professional culture, compounded by a lack of basic education on risk and risk management, the lowlevel of citizen participation in politics, declining social ties within communities, and the profit-seeking behavior of business and industries which undermined the coupling of economy and society despite this being held onto in popular attitudes.

Less clear in any process of future change were the roles of civil society and business. To foster momentum for transition, some respondents pointed to business as a key stakeholder. The case for more involvement from business speaks to the closeness of social benefit and economic activity in the Japanese imagination. Business groups were expected to underpin social and economic activities and provide technical as well as tax resources to government. Although the profit motive is accepted, business is seen to have some strengths to offer future regimes: with its larger financial base and technical resource (Avenell 2016). As a model 
case, a scholar in urban planning pointed to the potential of area management in the business districts such as Marunouchi where community-based urban renewal projects involving business entities have included disaster management.

\section{Conclusion}

The disaster risk management regime in Tokyo has gradually expanded from resistance to include elements of resilience. Policy has increasingly promoted selfhelp and community-cooperation approaches within existing strategy that combines both development and disaster management.

Perhaps most striking in shaping potential for transition is the strength of established values and their institutionalization.

Popular values include:

- trust in authority and its technical expertise;

- a belief in the inseparability of economic and social wellbeing;

- a belief in a low inequality of Japanese society.

Professional values include:

- respect for hierarchy in science and policy;

- a desire to build policy from past experiences.

In response to past extreme events, the regime has undertaken largely reactive policy-making adjusted within a national policy framework and directed by the Governor of Tokyo. Continued high public trust in government limits spontaneous, local innovation that might give rise to alternative risk management priorities or practices. Trust may be undermined if inequality and poverty continue to grow revealing a break in received notions of coupled economic and social wellbeing.

Under this current administrative regime, structural challenges to transition include the slow take-up of forward looking climate risk assessments and a culture of policy-making that is more comfortable with historical hazard and risk assessment methodologies associated with earthquake risk management. Common administrative practices relying on sectoral divisions and relatively rigid budget allocation also make it difficult for mid- and senior-level administrators to innovate more joined-up approaches to risk management and development planning. Regular rotation of administrative personnel impedes institutional memory for effective reflection on longer-term trends, including emerging needs. While some external experts continuously pass context-specific knowledge down throughout the rotations, there are challenges to effectively draw on scientific and intellectual resources from outside TMG. Administration's customary reticence upon academic 
structures limits the diversity of science voices in policy-making, and price competition can prevent the best commercial consultancy input.

Though earthquake risks have been the major focus for risk management to date, climate risk has gained increasing attention over the past two decades. However, management continues to be framed by past events rather than projected risk, and it is the reproduction of this outlook through administrative structure and governance culture that characterize Tokyo's constrained operating room for risk management and potential for transition.

\section{Acknowledgments}

Research reported in this paper was undertaken as part of the Belmont Forum funded Transformation and Resilience on Urban Coasts (TRUC) project. This project was supported by Japan Society for the Promotion of Science, the UK Natural Environment Research Council and Economic and Social Research Council (NE/L008971/1) the German Research Foundation (GZ: BI 1655/1-1), The Ministry of Earth Sciences, Government of India (MoES/01-CZM/Truc/2013) and US National Science Foundation (ICER-1342966).

\section{References}

Abe, AK (2011). The Myth of egalitarian society: poverty and social exclusion in Japan. In: P Saunders and R Sainsbury (eds.) Social Security, Poverty and Social Exclusion in Rich and Poorer Countries, Mortsel: Intersentia Publishing, pp. 175-199.

Aiba, S, Ichiko T and Nakabayashi I (2007). Pre-disaster measures for post-disaster recovery planning from the next Tokyo earthquake: Through earthquake disaster recovery measures and its drills with communities. Journal of Geography, 116(3/4): 557-575. (in Japanese).

Aldrich, DP (2008). Site Fights: Divisive Facilities and Civil Society in Japan and the West. Ithaca: Cornell University Press.

Aldrich, DP (2011). The power of people: social capital's role in recovery from the 1995 Kobe earthquake. Natural Hazards, 56(3): 595-611.

Aldrich, DP (2012). Post-crisis Japanese nuclear policy: From top-down directives to bottom-up activism. Asia Pacific Issues, 103, 1-12.

Aldrich, DP (2013). Rethinking civil society-state relations in Japan after 3/11. Polity, 45(2): 249-264.

Aldrich, DP (2016). It's who you know: Factors driving recovery from Japan's 11 March 2011 disaster. Public Administration, 94(2): 399-414.

Aldrich, DP and Meyer M (2015). Social capital and community resilience. American Behavioral Scientist, 59(2): 254-69.

Aldrich, DP and Sawada Y (2015). The physical and social determinants of mortality in the 3.11 tsunami. Social Science and Medicine, 124: 66-75. 
Aoki, K (2010). Policy process analyses of the Japanese local governments' progressive low carbon measures: Based upon the findings obtained from multiple case studies. The Fudai Keizai Ronshu: The Journal of Economic Studies, Toyama University, 56(2): 337-371. (in Japanese).

Aoki, K and Motoki Y (2007). A policy process analysis of the Tokyo Metropolitan Government's global warming prevention measures: Importance and implications of the policy dynamics emerging from the progressive local initiatives. The Fudai Keizai Ronshu: The Journal of Economic Studies, Toyama University, 53(2): 247-297. (in Japanese).

Avenell, S (2015). Transnationalism and the evolution of post-national citizenship in Japan. Asian Studies Review, 39(3): 375-394.

Avenell, S (2016). Antinuclear radicals: Scientific experts and antinuclear activism in Japan. Science Technology and Society, 21(1): 88-109.

Baba, K (2010). Effectiveness and diffusion of local climate policy: A case of GHG emission reporting system. Energy and Resources, 31(2): 1-9. (in Japanese).

Fire and Disaster Management Agency (2016). Heisei 27 Nendo Shobo Hakusho (Fiscal Year 2015 White Paper on Extinguishing Fire). http://www.fdma.go.jp/html/ hakusho/h27/h27/index3.html [October 31, 2016]. (in Japanese).

Government of Japan (2015). National Plan for Adaptation to the Impacts of Climate Change. http://www.env.go.jp/earth/ondanka/tekiou/siryo1.pdf [August 16, 2016]. (in Japanese).

Hasegawa, K, Shinohara C and Broadbent J (2007). The effect of 'social expectation' on the development of civil society in Japan. Journal of Civil Society, 3(2): 179-203.

Hijioka, Y, Takano S, Oka K, Yoshikawa M, Ichihashi A, Baba K and Ishiwatari S (2016). Potential of existing policies of the Tokyo Metropolitan Government for implementing adaptation to climate change. Regional Environmental Change, 16(4): 967978.

Hommerich, C (2012). Trust and subjective well-being after the Great East Japan Earthquake, Tsunami and Nuclear Meltdown: Preliminary results. International Journal of Japanese Sociology, 21: 46-64.

Iwasa, M (2008). A viewpoint of culture of disaster: Self-, mutual- and public-help. Journal of Liberal Arts, Seijoh University, 4: 5-13. (in Japanese).

Jones, RS (2007). Income Inequality, Poverty and Social Spending in Japan. OECD Economics Department Working Papers, No. 556, OECD publishing.

Nakagawa, Y and Shaw R (2004). Social capital: A missing link to disaster recovery. International Journal of Mass Emergencies and Disasters, 22(1): 5-34.

Ministry of the Environment (2014). Environmental Health Manual on Heat Strokes. http://www.wbgt.env.go.jp/heatstroke_manual.php [August 16, 2016]. (in Japanese).

Ministry of Health, Labour and Welfare (2011). Fiscal Year of 2012 White Paper on Health, Labour and Welfare. http://www.mhlw.go.jp/wp/hakusyo/kousei/12/ [August 16, 2016]. (in Japanese).

Mitchell, JK (1999). Crucibles of Hazard: Mega-Cities and Disasters in Transition. Tokyo: United Nations University Press.

Nakabayashi, I (2008). Earthquake vulnerability assessment and anti-earthquake disaster city planning in Tokyo. Journal of Urban Science, (2): 69-81. (in Japanese). 
Numata, M, Kondo S, Inoue M and Meguro K (2011). Comparative analysis of Regional Disaster Prevention Plan for regional cooperation framework. Seisan Kenkyu, 63(6): 755-763. (in Japanese).

OECD (2014). Society at a Glance 2014: OECD Social Indicators. OECD Publishing. http://dx.doi.org/10.1787/soc_glance-2014-en [August 16, 2016].

Ogawa, A (2004). Invited by the state: Institutionalizing volunteer subjectivity in contemporary Japan. Asian Anthropology, 3: 71-96.

Onishi, T (2010). Teitanso-Shakai ni Machete Machizukuri (City planning towards towards a low-carbon society). In: T Onishi and H Kobayashi (eds.) Teitanso-Toshi: Korekara no Machizukuri (Low Carbon City: Future Urban Planning) Kyoto: Gakukgei Shuppansha, pp. 8-29. (in Japanese).

Oono, T (2010). Teitanso-Toshi eno Tenkan wo Mezasu Tokyo no Torikumi (Actions of Tokyo aiming at transition towards a low-carbon city). In: T Onishi and H Kobayashi (eds.) Teitanso-Toshi: Korekara no Machizukuri (Low Carbon City: Future Urban Planning), Kyoto: Gakukgei Shuppansha, pp. 179-197. (in Japanese).

Pantazis, C (2014). Introduction: Comparative perspectives on poverty and inequality: Japan and the United Kingdom. Social Policy and Society, 13(1): 63-67.

Samuels, R (2013). 3.11: Disaster and Change in Japan. Ithaca, NY: Cornell University Press.

Sassen, S (2013). Global City: New York, London, Tokyo (Revised Edition). Princeton: Princeton University Press.

Seidensticker, E (1983). Low City, High City: Tokyo from Edo to the Earthquake. New York: Alfred A. Knopf.

Seidensticker, E (1990). Tokyo Rising: The City since the Great Earthquake. New York: Alfred A. Knopf.

Shirai, N and Baba K (2014). The situation and barriers on the implementation of climate change adaptation in local governments of Japan. Environmental Science, 27(5): 324-334. (in Japanese).

Shirai, N and Tanaka M (2015). Analysis of ideal directions of climate change adaptation and problems in implementing them for local Japanese governments. Journal of Disaster Research, 10(3): 420-428.

Solecki, W, Pelling M and Garschagen M (2017). Transitions between risk management regimes in megacities, Ecology and Society, in review.

Tanaka, M, Shirai N, Yamamoto T and Kimura H (2011). Issues and trends on global warming adaptation policy in Japanese local governments. Proceedings of 39th Annual Meeting of Environmental Systems Research, Japan Society of Civil Engineers, pp. 309-314. (in Japanese).

TMG (2011). Tokyo-To Bosai Taio Shishin (Tokyo Metropolitan Government Disaster Response Guidelines). http://www.bousai.metro.tokyo.jp/taisaku/1000061/1000368. html [August 16, 2016]. (in Japanese).

TMG (2014a). The Long-Term Vision for Tokyo. http://www.seisakukikaku.metro.tokyo.jp/ tokyo_vision/vision_index/ [August 16, 2016].

TMG (2014b). Chiiki Bosai Keikaku (Tokyo Local Plan for Disaster Prevention). http:// www.bousai.metro.tokyo.jp/taisaku/1000061/1000903/index.html [August 16, 2016]. (in Japanese). 
TMG (2014c). Tokyo no Bosai Puran (Tokyo Disaster Prevention Plan). http://www. bousai.metro.tokyo.jp/taisaku/1000061/1001592.html [August 16, 2016]. (in Japanese).

TMG (2015). Koreisha no Kyoju-Antei Kakuho Puran (Plan for Securing Stable Housing for the Aging Population). http://www.metro.tokyo.jp/INET/KEIKAKU/2015/03/ 70p3r200.htm [August 16, 2016]. (in Japanese).

TMG (2016a). Statistics of Tokyo. http://www.toukei.metro.tokyo.jp/jsuikei/js-index.htm [August 16, 2016]. (in Japanese).

TMG (2016b). Heisei 28 Nendo Tokyo-To no Yosan-An no Gaiyo (The Budget Plan of Tokyo Metropolitan Government for the Fiscal Year of 2016). http://www.zaimu. metro.tokyo.jp/syukei1/zaisei/20160210_heisei28nendo_tokyotoyosanangaiyou/ 28nendoyosanannogaiyou.pdf [August 16, 2016]. (in Japanese).

United Nations, Department of Economic and Social Affairs, Population Division (2015). World Urbanization Prospects: The 2014 Revision, (ST/ESA/SER.A/366). https:// esa.un.org/unpd/wup/Publications/Files/WUP2014-Report.pdf [August 16, 2016].

Veszteg, R, Y Funaki and A Tanaka (2014). The impact of the Tohoku earthquake and tsunami on social capital in Japan: Trust before and after the disaster. International Political Science Review, 36(2): 119-138. 